\title{
VAGINAL CANCER WITH UTERINE PROLAPSE: A RARE ENTITY
}

M. Bouhani1, I. zemni1, I. bouraoui1, M. slimene1, J. ben hassouna1, M. hechiche1, R. chargui1, K. rahal1

1Salah Azaiz Institute, Oncologic Surgery, Tunis, Tunisia

\section{Introduction:}

Primary vaginal cancer is a rare disease of the female gynecological tract and mainly affects postmenopausal women. It accounts for less than $0.5 \%$ of female cancers.

Uterine prolapse combined with vaginal cancer is an extremely rare condition. Until now, only less than 20 cases of primary vaginal cancer complicated by uterine prolapse have been reported. Squamous cells carcinoma is the most common type in this entity.

\section{Methods:}

We present 3 cases of primary vaginal cancer in women with uterine prolapse treated in Salah Azaiez institute between 1997 and 2017

\section{Results:}

The mean age was 74.6 year, the mean parity was 6 parity per woman. Symptoms were blood stained discharge, foul odor leukorrhea, and severe pelvic pain for the last 1 to 3 months. The mean tumor size was 6.3 $\mathrm{cm}$ and the tumor location was on the anterior wall in 2 cases and the posterior wall in one case. The prolapse was reduced under intravenous sedation in operating room. On gynecologic examination, uterus was normal in size, no adnexal mass was examined, one patient presented with vesico-vaginal fistulea. There was no inguinal lymph node.

Biopsy of the ulcer at vaginal wall revealed invasive squamous cell carcinoma of vagina. The extention work up didn't reveal any metastasis in none of patients.

The tumor was staged at stage I of FIGO in 2 patients and at stage IVA of FIGO in one patient.

All the patients underwent surgery.

Surgery consists on a total hysterectomy, bilateral salpingo-oophorectomy, bilateral pelvic lymph node dissection, and total vaginectomy in two patients and anterior pelvic exenteration in one patient. they didn't have a reconstitution of the vaginal wall. No complication after surgery was noted.

The margins were free of disease.

Two patients had adjuvant radiotherapy. The mean follow up was 37.3 months and the patients were free of disease during follow up period.

\section{Discussion:}

Uterine prolapsed combined with vaginal cancer is an extremely rare condition. Until now, only less than 20 cases of primary vaginal cancer complicated by uterine prolapse have been reported.

Great multiparity, postmenopausal status, also chronic cough, obesity, and chronic constipation are known as risk factors for uterine prolapse. Moreover, multiparity, postmenopausal status, and prolapse also represent risk factors for the development of vaginal cancer. Hence, early sexual intercourses, Human Papilloma Virus (HPV) infection, chronic vaginal irritation are also risk factors for vaginal cancer

Due to the rarity of this entity, there are very few publications on the subject, and it is essentially unique cases that are reported. There is no consensus on the appropriate treatment of these cancers on genital prolapse. In our cases, treatment guidelines have been extrapolated from studies of vaginal carcinoma without uterine prolapse.

For most stage I, radiation therapy is used in squamous cell vaginal cancer. However, if the tumor is located is in the upper vagina, it can be treated with radical hysterectomy, removal of bilateral radical pelvic lymph nodes, or radical or partial vaginectomy. The tumor located in the lower third of vagina length may benefit also of partial vaginectomy with colporrhaphy. Postoperative external beam radiation can be used to treat small deposits of cancer cells that have spread to pelvic lymph nodes.

The usual treatment for stages II, III, or IV is radiation therapy, and chemotherapy may be combined with radiation helping it work better. In, those cases salvage surgery may be discussed on a case by case basis.

\section{Conclusion:}

Uterine prolapse combined with vaginal cancer is a very uncommon condition. Our series seems to be important due to number of cases reported and the successful treatment management. 